\title{
Influencia del contenido de finos del hormigón en la respuesta estructural a flexión y cortante de vigas de hormigón armado
}

\section{Influence of the fines content in the flexural and shear structural response of reinforced concrete beams}

D. Ferreira ${ }^{(*)}, \underline{\text { E. Oller }}^{(*)}$, J. M. Bairán ${ }^{(*)}$, S. Carrascón ${ }^{(* *)}$, A. Marí $^{(*)}$

\section{RESUMEN}

Este artículo presenta un estudio experimental sobre el comportamiento a flexión y cortante de vigas de hormigón armado con un contenido de finos $\left(275 \mathrm{~kg} / \mathrm{m}^{3}\right)$ superior al límite permitido por la Instrucción EHE-o8 $\left(175 \mathrm{~kg} / \mathrm{m}^{3}\right)$. En el rango estudiado, el contenido de finos apenas afectó a la respuesta a flexión. La resistencia a cortante de vigas con alto contenido de finos fue menor que la de las vigas de hormigón convencional, en especial para las vigas con cuantía alta de armadura longitudinal y con armadura de cortante, con una reducción de hasta el 23 \% en la carga última. Esta reducción se considera debida a la disminución de propiedades mecánicas del hormigón que afecta especialmente al cortante resistido por fricción en la fisura. Por último, los ensayos realizados han permitido verificar un modelo numérico de análisis no lineal evolutivo para predecir el comportamiento de estructuras de hormigón con distintos contenidos de finos.

Palabras clave: Contenido de finos; árido; hormigón armado; cortante; flexión.

\section{ABSTRACT}

This paper presents an experimental study on the flexural and shear behavior of reinforced concrete beams with a fines content $\left(275 \mathrm{~kg} / \mathrm{m}^{3}\right)$ higher than the limit value allowed by the Spanish Concrete Code EHE-o8 $\left(175 \mathrm{~kg} / \mathrm{m}^{3}\right)$. In the studied range, the fines content scarcely affected the flexural response. The shear strength of the beams with a high content of fines was lower than that of the beams cast with conventional concrete, especially for those with a high longitudinal internal reinforcement ratio and with transversal stirrups, where the reduction of the ultimate load was up to $23 \%$. Such a reduction is considered due to the reduction of mechanical properties of concrete and that of the aggregate interlock along the crack length. Finally, the experimental results have allowed verifying a numerical model for the non-linear analysis of RC members to accurately predict the influence of the amount of fine aggregate on the structural response.

Keywords: Fines content; aggregate; reinforced concrete; shear; bending.

(*) Universitat Politècnica de Catalunya, Barcelona (España).

${ }^{(* *)}$ Instituto Español del Cemento y sus Aplicaciones, Madrid (España).

Persona de contacto/Corresponding author: eva.oller@upc.edu (E. Oller).

Cómo citar este artículo/Citation: Ferreira, D., Oller, E., Bairán, J. M., Carrascón, S., Marí, A. (2015). Influencia del contenido de finos del hormigón en la respuesta estructural a flexión y cortante de vigas de hormigón armado. Informes de la Construcción, 67(539): e097, doi: http://dx.doi.org/10.3989/ic.14.039.

Licencia / License: Salvo indicación contraria, todos los contenidos de la edición electrónica de Informes de la Construcción se distribuyen bajo una licencia de uso y distribución Creative Commons Reconocimiento no Comercial 3.o. España (cc-by-nc). 


\section{INTRODUCCIÓN}

De acuerdo con la Instrucción del Hormigón Estructural EHE-o8 (1), se entiende por finos aquellos áridos que pasan por el tamiz 0,063. La presencia de estos finos incide tanto en el comportamiento reológico de la masa fresca de hormigón, como en las propiedades del mismo una vez endurecido. En estado fresco, un alto contenido de finos aumenta la trabajabilidad e implica una mayor demanda de contenido de agua que puede repercutir negativamente en el endurecimiento del hormigón. Sin embargo, ésta se puede reducir mediante la presencia de aditivos. En estado endurecido, las partículas de finos pueden formar una delgada capa sobre la superficie de los áridos más grandes, disminuyendo así su adherencia.

La vigente Instrucción EHE-o8 (1) limita el contenido de finos en el hormigón, resultante de sumar el contenido de partículas del árido grueso y del árido fino que pasan por el tamiz UNE o,063 y la componente caliza, en su caso, del cemento, a un valor inferior a $175 \mathrm{~kg} / \mathrm{m}^{3}$, que puede incrementarse a $185 \mathrm{~kg} / \mathrm{m}^{3}$ en el caso de emplear agua reciclada. Sin embargo, está ampliamente comprobado que en hormigones autocompactantes este límite de finos se sobrepasa holgadamente sin merma en sus propiedades en estado fresco, en las propiedades mecánicas en estado endurecido y en la durabilidad (2).

En base a lo anterior, el objetivo principal del presente trabajo es estudiar el comportamiento estructural de vigas de hormigón con un contenido de finos superior al permitido por la normativa, comparando los resultados con vigas fabricadas con un contenido de finos que no supere ese límite.

Este trabajo se ha realizado de forma paralela a otros estudios encargados por el Instituto Español del Cemento y sus Aplicaciones relativos al estado fresco y endurecido de hormigones convencionales con cantidad de finos superior a la permitida en la Instrucción EHE-08 (1), realizado por Sáez y Aguado (2) y a la durabilidad.

En concreto, Sáez y Aguado (2) realizaron un estudio de caracterización mecánica de dos tipos de hormigones con resistencia $25 \mathrm{~N} / \mathrm{mm}^{2}$ y con dos contenidos de finos (175 y $275 \mathrm{~kg} / \mathrm{m}^{3}$ ). En su trabajo concluyen que la dosificación con mayor contenido de finos presenta condiciones similares de trabajabilidad del hormigón fresco, menor resistencia mecánica y módulo de elasticidad, mayor pérdida de masa y fluencia. Fruto de este estudio se concluye que el límite de contenido de finos de la Instrucción EHE-08 (1) podría aumentarse hasta valores si- milares a los hormigones autocompactantes $\left(250 \mathrm{~kg} / \mathrm{m}^{3}\right)$ con ventajas desde el punto de vista de sostenibilidad.

En 2006, Katz y Baum (3) realizaron un estudio similar estudiando el efecto de contenido de finos de hasta $227 \mathrm{~kg} / \mathrm{m}^{3}$ en hormigones de resistencia $35 \mathrm{~N} / \mathrm{mm}^{2}$. En este caso llegaron a obtener una mejora de hasta el $30 \%$ de la resistencia a compresión del hormigón a 28 días aumentando el contenido de finos en la mezcla.

Vilanova et al. (4) realizaron una evaluación de los modelos existentes en la Instrucción EHE-o8 (1), Eurocódigo 2 (5) y ACI-o8 (6) para estimar las propiedades del hormigón autocompactante con elevado contenido de finos, concluyendo que todas ellas son aplicables al obtenerse ajuste altos, siendo los más precisos, los modelos del ACI-08 (6).

La mayor parte de estudios existentes se centran en la caracterización del material, siendo menores los estudios experimentales relativos al comportamiento estructural de elementos de hormigón armado con elevado contenido de finos, y casi todos ellos se refieren al empleo de hormigones autocompactantes (7), y no hormigones convencionales. Por ello, el objetivo principal de este trabajo es el estudio de la influencia del contenido de finos en la respuesta a flexión y a cortante de vigas de hormigón armado convencional.

\section{DESCRIPCIÓN DE LA CAMPAÑA EXPERIMENTAL}

Para alcanzar el objetivo mencionado anteriormente, se ensayaron 8 vigas simplemente apoyadas de hormigón armado bajo cargas puntuales, 4 de ellas con $175 \mathrm{~kg} / \mathrm{m}^{3}$ de contenido de finos y 4 de ellas con $275 \mathrm{~kg} / \mathrm{m}^{3}$, todas ellas con la misma resistencia característica $\left(f_{c k}=25 \mathrm{~N} / \mathrm{mm}^{2}\right)$.

\subsection{Ensayos de caracterización mecánica de los materiales}

Antes de los ensayos estructurales se realizaron ensayos de caracterización del hormigón sobre probetas cilíndricas de la misma amasada que las vigas, almacenadas en condiciones ambientales controladas y/o iguales a las vigas, a fin de poder comparar las predicciones teóricas con los resultados experimentales.

La Tabla 1 muestra los resultados de los ensayos normalizados de resistencia a compresión $\left(f_{c m}\right)$, a tracción indirecta $\left(f_{c t m}\right)$ y módulo de elasticidad $\left(E_{c m}\right)$ del hormigón sobre probetas cilíndricas a 7, 28 y 90 días (edad de ensayo de las vigas).

Tabla 1. Propiedades del hormigón fabricado con contenido de finos: 175 y $275 \mathrm{~kg} / \mathrm{m}^{3}$.

\begin{tabular}{|c|c|c|c|c|}
\hline $\begin{array}{l}\text { Contenido de } \\
\text { finos }\left(\mathrm{kg} / \mathrm{m}^{3}\right)\end{array}$ & $\begin{array}{c}\text { Edad } \\
\text { (días) }\end{array}$ & $\underset{\left(\mathrm{N} / \mathbf{m m}^{2}\right)}{\boldsymbol{f}_{\mathrm{mm}}}$ & $\underset{\left(\mathbf{N} / \mathbf{m m}^{2}\right)}{\boldsymbol{f}_{\mathrm{ctm}}}$ & $\underset{\left(\mathrm{N} / \mathbf{m m}^{2}\right)}{E_{\mathrm{mm}}}$ \\
\hline \multirow{4}{*}{175} & 7 & 19,68 & - & 27736 \\
\hline & 28 & 27,12 & 2,93 & 31078 \\
\hline & 90 & 31,34 & - & 32438 \\
\hline & $90^{*}$ & 31,16 & 3,23 & - \\
\hline \multirow{4}{*}{275} & 7 & $18,84(-4,3 \%)$ & - & $27578(-0,5 \%)$ \\
\hline & 28 & $24,40(-10,0 \%)$ & $2,58(-12,0 \%)$ & $29343(-5,5 \%)$ \\
\hline & 90 & $26,45(-15,6 \%)$ & - & $31123(-4,0 \%)$ \\
\hline & $90^{*}$ & $30,86(-0,9 \%)$ & $3,07(-4,95 \%)$ & - \\
\hline
\end{tabular}

* Probetas curadas en las mismas condiciones ambientales que las vigas. 
Se observa que, en todos los parámetros medidos, los resultados son algo menores en el hormigón con alto contenido en finos $\left(275 \mathrm{~kg} / \mathrm{m}^{3}\right)$ que en el hormigón convencional $(175 \mathrm{~kg} /$ $\mathrm{m}^{3}$ ), si bien las diferencias son mayores en las resistencias que en el módulo de elasticidad.

Se utilizó acero B50oS para las armaduras longitudinales y transversales de límite elástico garantizado $f_{y}=500 \mathrm{~N} / \mathrm{mm}^{2}$, carga unitaria de rotura $f_{u}=550 \mathrm{~N} / \mathrm{mm}^{2}$ y deformación bajo carga máxima $\varepsilon_{s u}=0,05$.

\subsection{Descripción de los ensayos}

Las vigas ensayadas eran de sección rectangular de ancho $225 \mathrm{~mm}$, canto $450 \mathrm{~mm}$, canto útil $400 \mathrm{~mm}$, y una longitud total de $5500 \mathrm{~mm}$. De las 8 vigas, 4 se ensayaron a flexión y 4 a cortante.

La nomenclatura empleada para los ensayos incluye información sobre:

- El tipo de ensayo: F (flexión), C (cortante).

- El contenido de finos: 175 o $275 \mathrm{~kg} / \mathrm{m}^{3}$

- La existencia o no de armadura transversal: S (sin) y T (con armadura transversal).

- La cuantía de armadura longitudinal del extremo ensayado: M (media), A (alta).

\subsubsection{Ensayos a flexión}

Se ensayaron dos vigas con una luz de 5,00 m entre apoyos bajo dos cargas puntuales situadas a 2,00 m de cada apoyo. El objetivo de estos ensayos fue observar si un elevado contenido de finos modificaba el comportamiento en servicio o rotura de las vigas.

La nomenclatura de las vigas ensayadas a flexión fue: F-175-T-M, F-175-T-A, F-275-T-M y F-275-T-A. Las vigas F-175-T-M y F-275-T-M se armaron longitudinalmente con $3 ø 16, \rho_{s}=0,0067$ y cercos $\varnothing 6$ a $150 \mathrm{~mm}$. En las vigas F-175-T-A y F-275-T-A se dispuso una cuantía más alta $\left(3 ø 25, \rho_{s}=0,0163\right)$ y cercos $\varnothing 8$ a $150 \mathrm{~mm}$ (ver Figura 1).

\subsubsection{Ensayos a cortante}

El objetivo de estos ensayos fue determinar si la cantidad de finos puede afectar al cortante resistido por el hormigón y a la resistencia a cortante por tracción en el alma. Esta componente depende tanto de la resistencia a tracción del hormigón como de la fricción entre caras internas de fisuras.

Cada viga se ensayó en dos ocasiones, una vez por cada extremo con una luz de 3,0 m, y bajo carga puntual situada a una distancia 1,2 $\mathrm{m}$ del apoyo (3 veces su canto útil). En los ensayos se produjo la rotura por cortante.
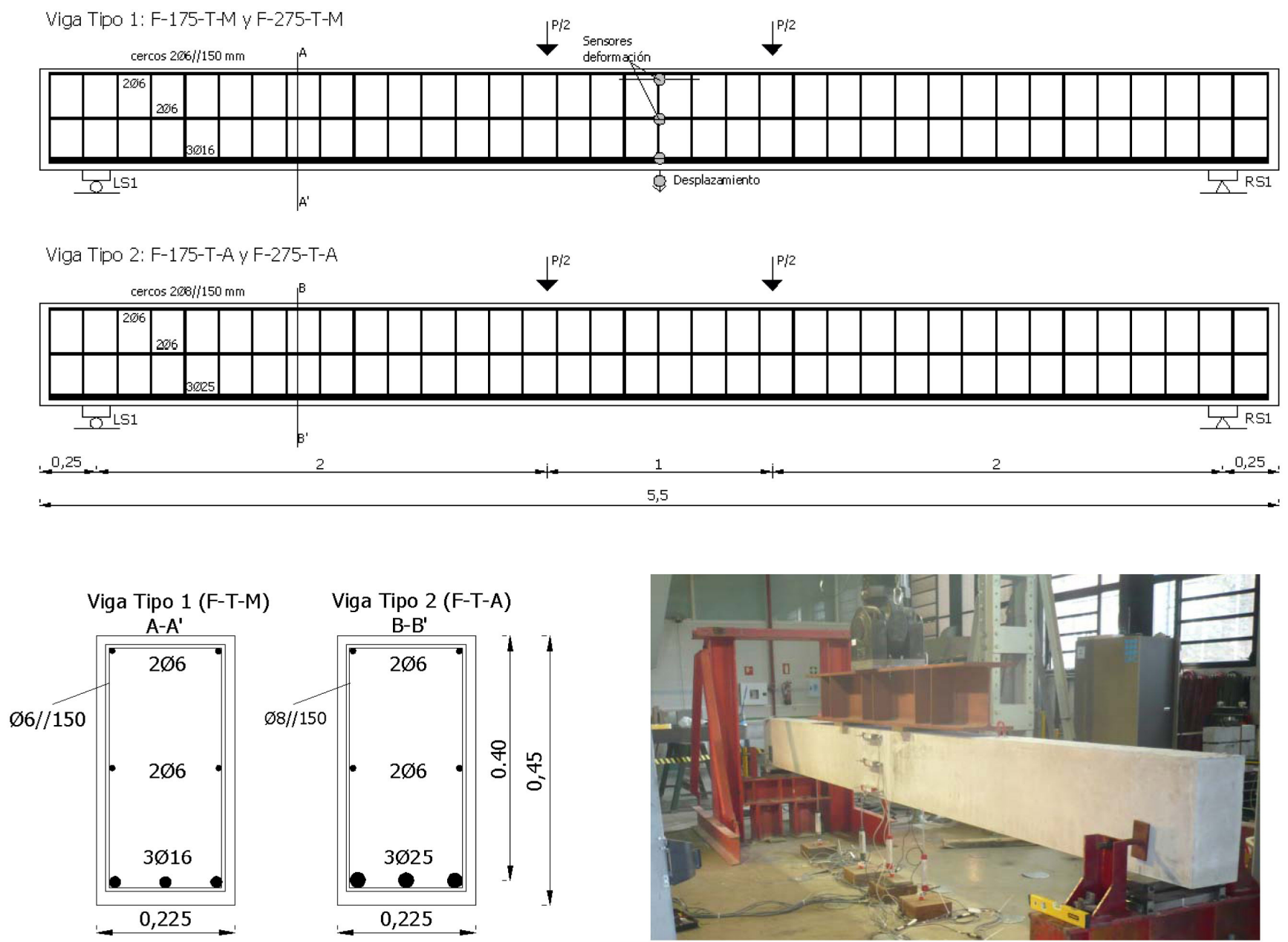

Figura 1. Geometría y esquema estático de los ensayos a flexión. 
La cuantía de armadura longitudinal de las vigas fue diferente en cada extremo. La armadura base a lo largo de toda la viga consistió en $3 ø 20$, y en uno de los extremos se dispuso de una armadura de refuerzo de $2 \varnothing 20$.

Para cada tipo de hormigón, se ensayaron dos vigas idénticas en cuanto a su geometría y a la armadura longitudinal de tracción: Viga 1, sin armadura transversal en la zona de rotura y Viga 2, con armadura transversal en dicha zona (ver Figura 2).

La armadura transversal consistió en cercos ø6/250 mm, en la zona con cuantía media de armadura longitudinal, en una longitud de 2,25 $\mathrm{m}$ de la viga desde el extremo. En los 2,25 m simétricos, se dispusieron $ø 6 / 200 \mathrm{~mm}$.

La nomenclatura de los ensayos de cortante fue: C-175-S-M, C-175-S-A, C-275-S-M, C-275-S-A, C-175-T-M, C-175-T-A, C275-T-M, C-275-T-A.

\subsection{Configuración de los ensayos e instrumentación}

Los aparatos de apoyo metálicos consistieron en una rótula fija y otra móvil. Las cargas puntuales fueron introducidas mediante un gato hidráulico con control de desplazamiento a una velocidad de $0,5 \mathrm{~mm} /$ minuto. Se realizó una precarga hasta un nivel del orden del $60 \%$ de la carga máxima estimada, con posterior descarga total, recarga hasta alcanzar la rama plástica de la curva carga-desplazamiento, descarga y de nuevo recarga hasta el agotamiento.

Se colocaron transductores de desplazamiento para medir los desplazamientos verticales y deformaciones medias y distorsiones angulares.

\section{RESULTADOS DE LAS CAMPAÑAS EXPERIMENTALES}

\subsection{Ensayos a flexión}

Tal y como se observa en la Figura 3, las curvas carga-desplazamiento son muy similares para las vigas con la misma cuantía de armadura longitudinal y con los dos contenidos de finos. La carga última es prácticamente idéntica independientemente del contenido de finos (Tabla 2). Los desplazamientos son muy similares para ambos contenidos de finos, aunque las vigas con mayor contenido de finos tienen menor resistencia a tracción y módulo de elasticidad del hormigón.

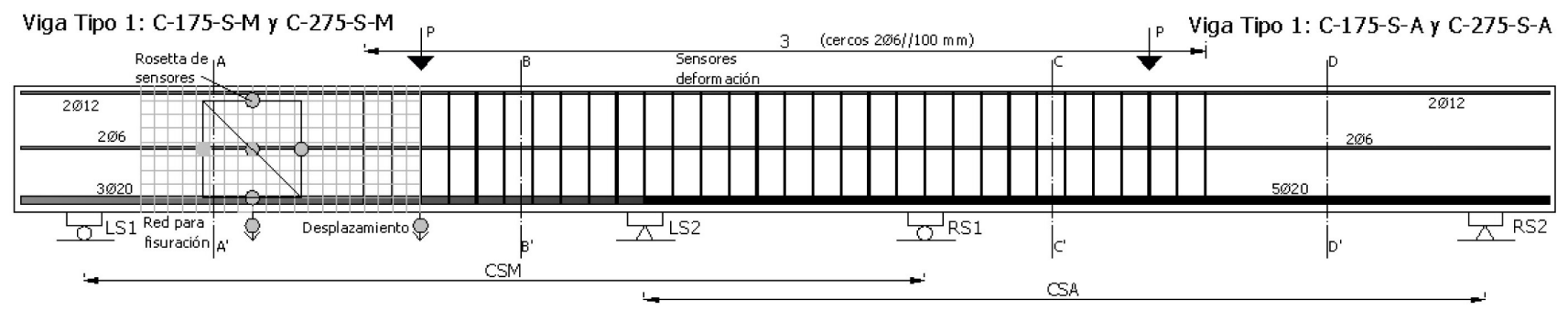

Viga Tipo 1: C-175-T-M y C-275-T-M
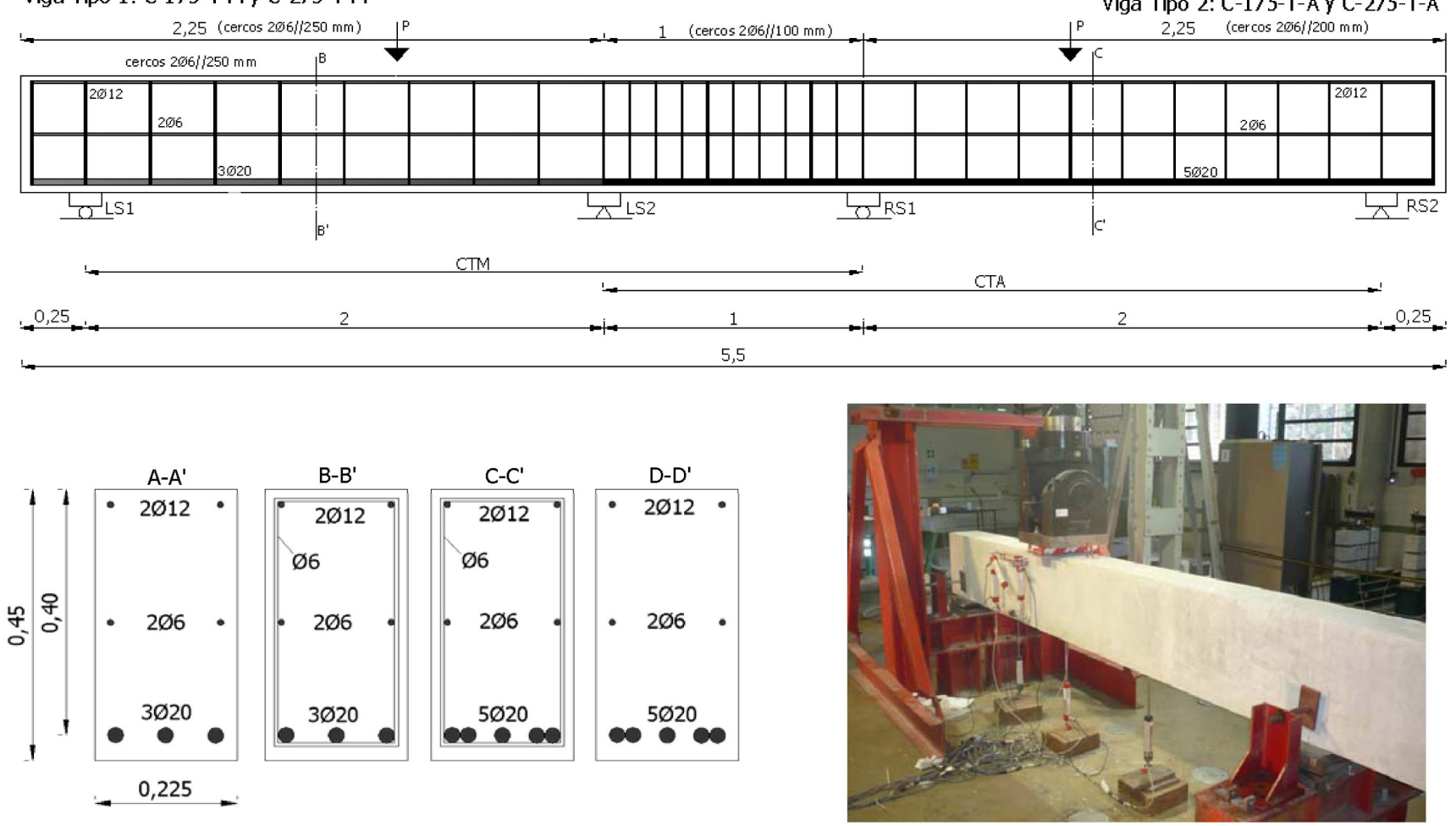

Figura 2. Geometría y esquema estático de los ensayos a cortante. 
En la Tabla 2 se presenta la separación media y máxima entre fisuras, el ancho medio de fisuras $\mathrm{w}_{\mathrm{m}} \mathrm{y}$ la flecha $\delta$ bajo carga de servicio. Se considera como carga de servicio, $50 \mathrm{kN}$ para las vigas de cuantía media y $120 \mathrm{kN}$ para las vigas de cuantía alta de armadura longitudinal. No se encuentran diferencias significativas en relación a la flecha en servicio entre los dos tipos de hormigón. La separación media entre fisuras suele disminuir para hormigón con mayor contenido de finos. Se constata que el ancho de fisuras en el hormigón con más contenido de finos es siempre igual o menor que en el convencional, lo que junto con la menor separación entre fisuras podría indicar una mejora de la adherencia, para el hormigón con alto contenido de finos.

La Figura 4 muestra la carga-deformación en la sección centro luz a la altura de la armadura longitudinal. Para las vigas con cuantía media se observa un comportamiento más dúctil para el caso de mayor contenido de finos. Sin embargo, dicha tendencia no se llega a observar en la viga con mayor cuantía de armadura longitudinal, debido a fallo de la instrumentación durante el registro de las deformaciones.

Tabla 2. Carga última en rotura, separaciones media y máxima entre fisuras, anchos de fisura y flechas bajo carga de servicio.

\begin{tabular}{|l|c|c|c|c|c|c|c|}
\hline \multirow{2}{*}{$\begin{array}{c}\text { Ensayos } \\
\text { experimentales }\end{array}$} & \multicolumn{2}{|c|}{ Rotura } & \multicolumn{4}{c|}{ Servicio } \\
\cline { 3 - 8 } & $\mathbf{P}_{\mathbf{u}}$ & $\begin{array}{c}\text { Modo de } \\
\text { rotura }\end{array}$ & $\begin{array}{c}\mathbf{s}_{\mathbf{m}} \\
(\mathbf{m m})\end{array}$ & $\begin{array}{c}\mathbf{S}_{\text {max }} \\
(\mathbf{m m})\end{array}$ & $\begin{array}{c}\mathbf{w}_{\mathbf{m}} \\
(\mathbf{m m})\end{array}$ & $\begin{array}{c}\boldsymbol{\delta} \\
(\mathbf{m m})\end{array}$ \\
\hline \multirow{2}{*}{ F-T-A } & 175 & 296 & Flexión & 152 & 190 & 0,15 & 11,50 \\
\cline { 2 - 8 } & 275 & 290 & Flexión & 150 & 270 & 0,15 & 12,00 \\
\hline \multirow{2}{*}{ F-T-M } & 175 & 139 & Flexión & 152 & 190 & 0,16 & 8,76 \\
\cline { 2 - 8 } & 275 & 137 & Flexión & 138 & 240 & 0,14 & 8,80 \\
\hline
\end{tabular}

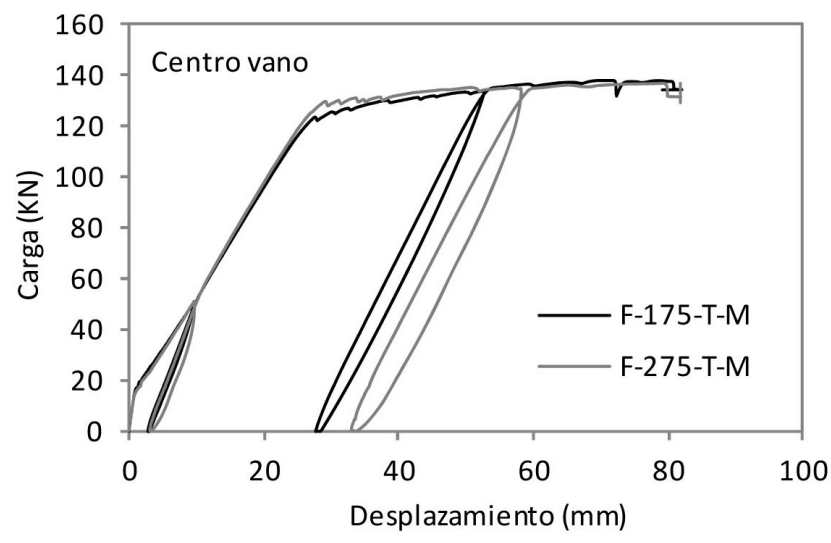

a) cuantía media de armadura longitudinal

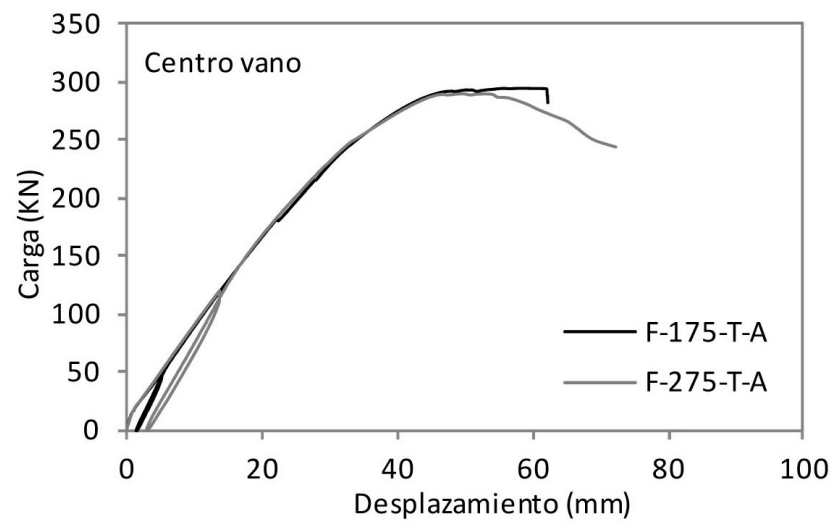

b) cuantía alta de armadura longitudinal

Figura 3. Carga-desplazamiento centro vano para los ensayos a flexión.

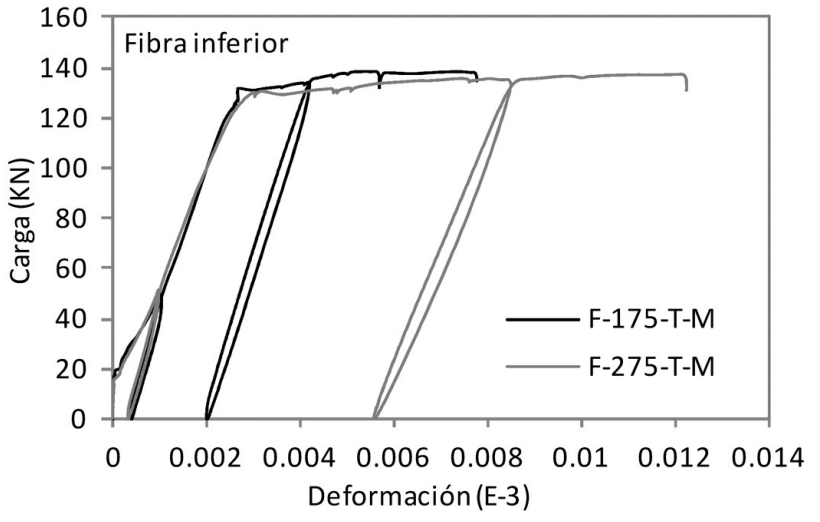

a) cuantía media de armadura longitudinal

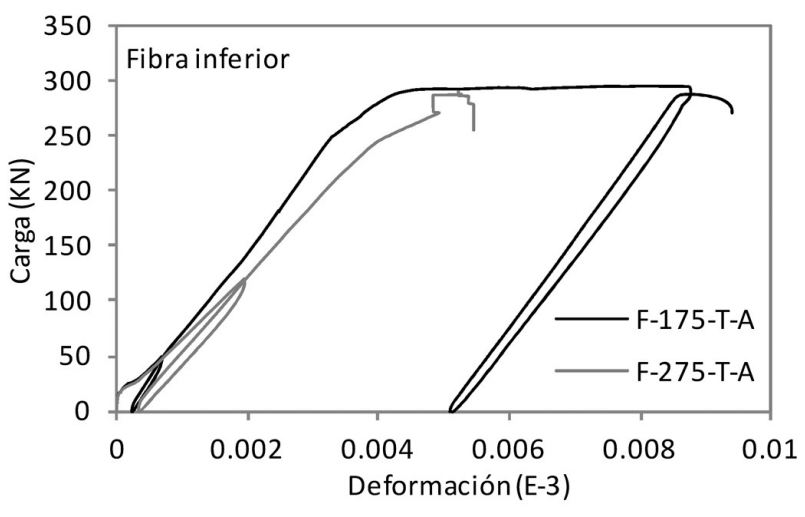

b) cuantía alta de armadura longitudinal

Figura 4. Carga-deformación en la sección de hormigón a la altura de la armadura longitudinal en el centro de la luz para las vigas ensayadas a flexión. 
La Figura 5 muestra los patrones de fisuración experimentales para ambas cuantías de armadura longitudinal. Las diferencias en relación al contenido de finos no parecen significativas en el caso de la cuantía media de armadura longitudinal. Se observa una mayor fisuración en el caso de mayor contenido de finos para las vigas armadas con mayor cuantía longitudinal. La separación media entre fisuras es inferior o igual para el caso de vigas con mayor cuantía de finos, por ello los anchos de fisuras son también menores, lo que está relacionado con una mejora en la adherencia.

\subsection{Ensayos a cortante}

\subsubsection{Vigas sin armadura transversal}

La Figura 6 presenta la curva carga-desplazamiento bajo el punto de aplicación de la carga para las vigas con cuantía media y con cuantía alta de armadura longitudinal.

La viga C-175-S-A alcanza una carga última de $181,1 \mathrm{kN}$ $\left(V_{u, \exp }=108,6 \mathrm{kN}\right)$, sin embargo, la viga $\mathrm{C}-275-\mathrm{S}-\mathrm{A}$, con $275 \mathrm{~kg} / \mathrm{m}^{3}$ de finos, resiste una carga inferior, de $158,4 \mathrm{kN}$ $\left(V_{u, \text { exp }}=95,0 \mathrm{kN}\right)$. Se observa una reducción de la capacidad resistente a cortante del orden del 12,5\% de la viga C-275-S-A respecto de la $\mathrm{C}-175-\mathrm{S}-\mathrm{A}$.
Para las vigas con cuantía media de armadura longitudinal, la capacidad resistente apenas se ve afectada por el contenido de finos. La carga última de la viga C-275-S-M es de $141 \mathrm{kN}\left(V_{u, \exp }=84,6 \mathrm{kN}\right)$ frente a $145,4 \mathrm{kN}\left(V_{u, \exp }=87,2 \mathrm{kN}\right)$ de la viga $\mathrm{C}-175-\mathrm{S}-\mathrm{M}$, es decir apenas un $3,0 \%$ de reducción.

Esta reducción de la capacidad resistente a cortante puede estar relacionada con la menor resistencia a tracción del hormigón y una posible disminución del engranamiento de los áridos al ser más suaves las caras de la fisura (7) (8).

Tal y como se observa en la Figura 6, para el caso de cuantía media de armadura longitudinal, la gráfica carga-desplazamiento es prácticamente independiente del contenido de finos. Sin embargo, para el caso de cuantía alta de armadura longitudinal, la viga con contenido alto de finos parece tener un comportamiento un poco menos rígido.

\subsubsection{Vigas con armadura transversal}

De forma similar al apartado anterior, la Figura 7 muestra la relación carga-desplazamiento para las vigas con armadura transversal.

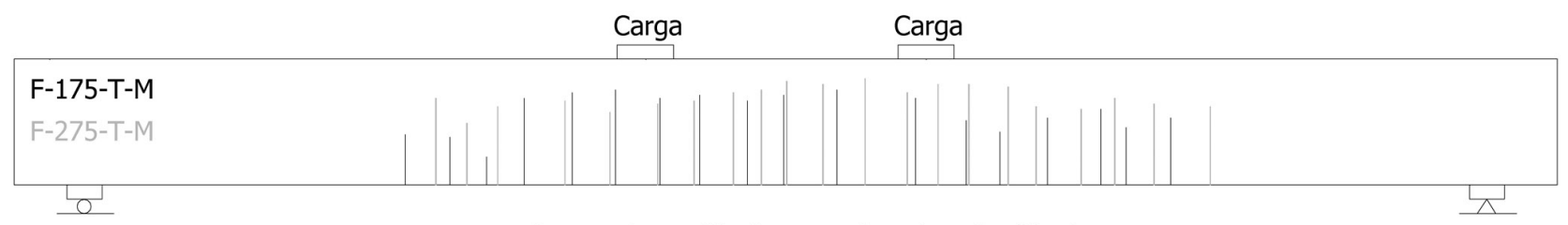

a) cuantía media de armadura longitudinal

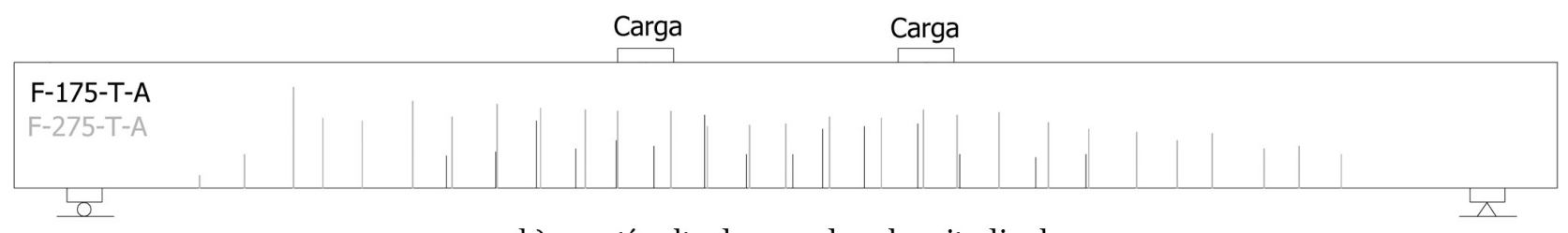

b) cuantía alta de armadura longitudinal

Figura 5. Patrones de fisuración de los ensayos de flexión.

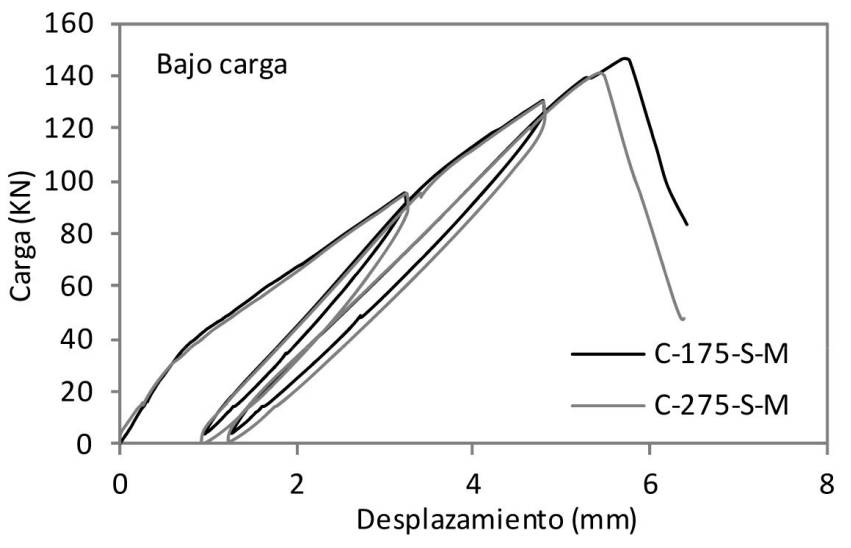

a) cuantía media armadura longitudinal sin estribos

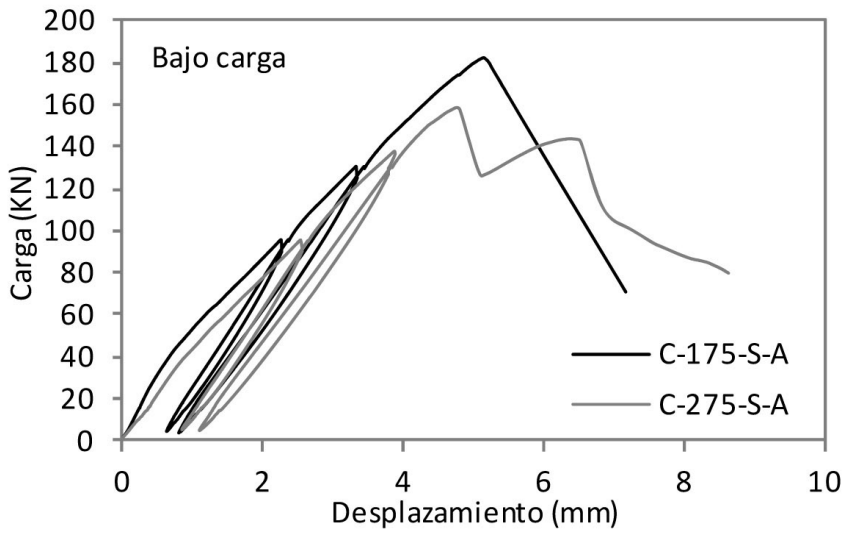

b) cuantía alta armadura longitudinal sin estribos

Figura 6. Carga-desplazamiento bajo carga. 


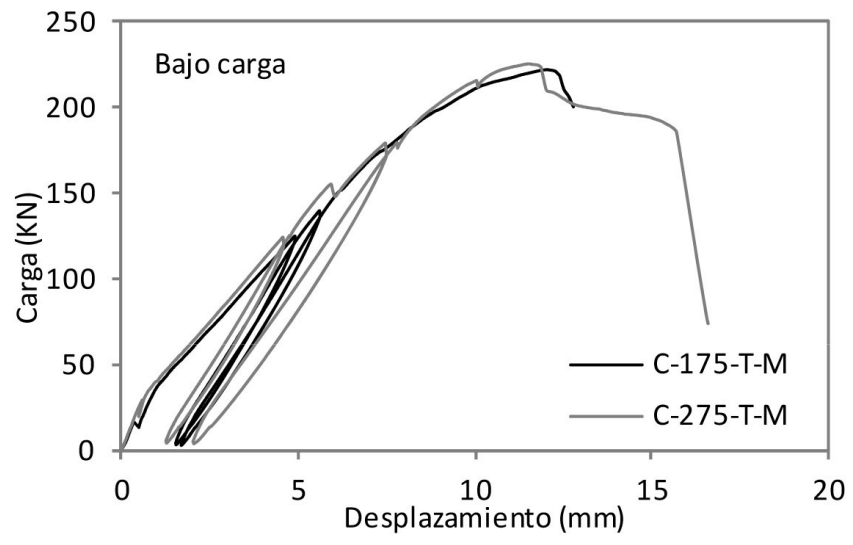

a) cuantía media armadura longitudinal con estribos

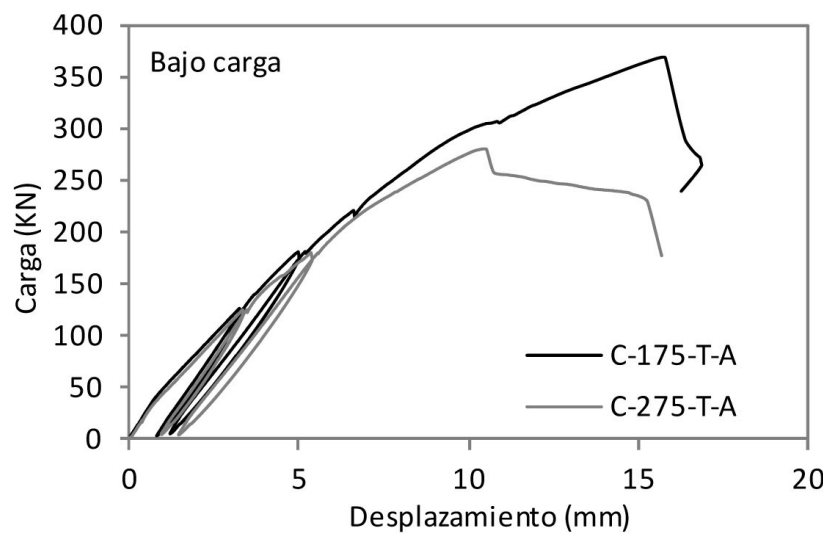

b) cuantía alta armadura longitudinal con estribos

Figura 7. Carga-desplazamiento bajo carga.

Para las vigas con cuantía alta de armadura longitudinal, se observa una diferencia considerable entre la capacidad resistente a cortante de las vigas con distinto contenido de finos. Mientras la viga de hormigón convencional, C-175-T-A resiste $367,3 \mathrm{kN}$ de carga última $\left(V_{u, \exp }=220,4 \mathrm{kN}\right)$, la viga C-275-T-A resiste $284 \mathrm{kN}\left(V_{u, \exp }=170,4 \mathrm{kN}\right)$, es decir un $22,7 \%$ menos.

En la curva carga-desplazamiento de la viga C-175-T-A se observa que tras alcanzar una pendiente prácticamente horizontal para una carga de $300 \mathrm{kN}$, se produce un incremento de rigidez y un aumento de la carga última, hasta los $367,3 \mathrm{kN}$. El mismo efecto, incluso más acusado, se observa en la curva carga-distorsión. Este fenómeno puede ser debido a un cambio de esquema resistente, se observa la formación de una fisura de cortante menos vertical, con un ángulo de inclinación menor de $30^{\circ}$, que cruza las fisuras existentes como se observa en la Figura 9, y que probablemente descarga las armaduras transversales.

Para las vigas con cuantía media de armadura longitudinal, se observó un comportamiento muy similar independiente-

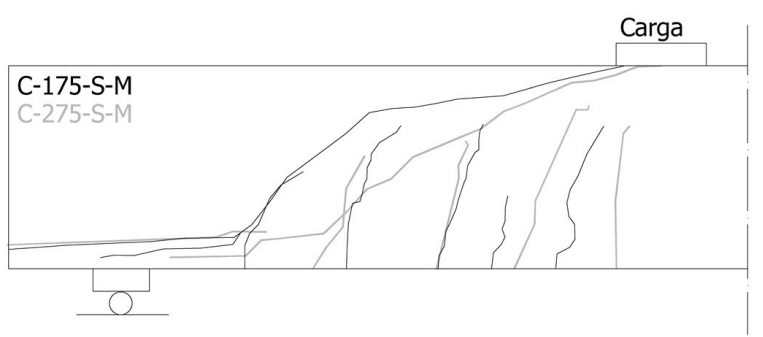

a) cuantía media armadura longitudinal sin estribos

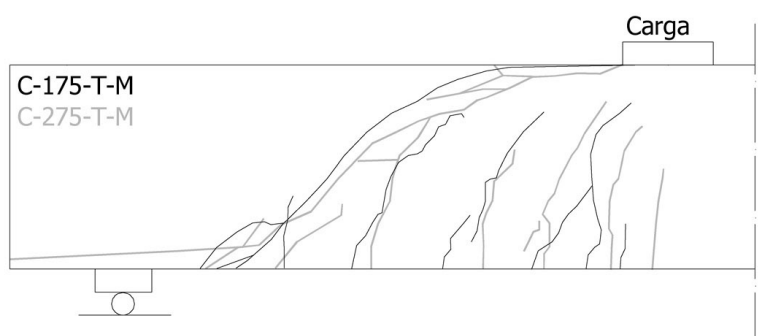

c) cuantía media armadura longitudinal con estribos mente del contenido de finos, obteniéndose un ligero aumento de capacidad portante de las vigas con $275 \mathrm{~kg} / \mathrm{m}^{3}$ de finos de apenas el 2,2 \% (225 kN frente a $220,1 \mathrm{kN})$.

Los patrones de fisuración de los ensayos a cortante de vigas con o sin armadura transversal, con cuantías medias y altas de armadura longitudinal y distinto contenido de finos se recogen en la Figura 8. Para los casos sin armadura de cortante, se observa que los patrones de fisuración son muy similares independientemente del contenido de finos. La única diferencia significativa se da para la cuantía media de armadura longitudinal, (Figura 8a) donde la fisura de cortante crítica se produce ligeramente desplazada hacia el punto de aplicación de la carga, con una segunda rama menos horizontal que para el caso con menor contenido de finos. Para las vigas con armadura de cortante, los patrones de fisuración son bastante similares y no se destacan diferencias relevantes en función del contenido de finos. Se observa cómo la existencia de armadura de cortante incrementa el número de fisuras y la inclinación de las fisuras con la directriz de la pieza. Sin embargo, la cuantía de armadura longitudinal prácticamente no influye en el patrón de fisuración.

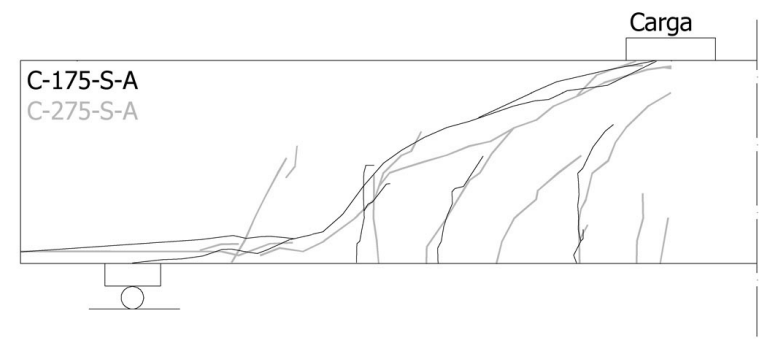

b) cuantía alta armadura longitudinal sin estribos

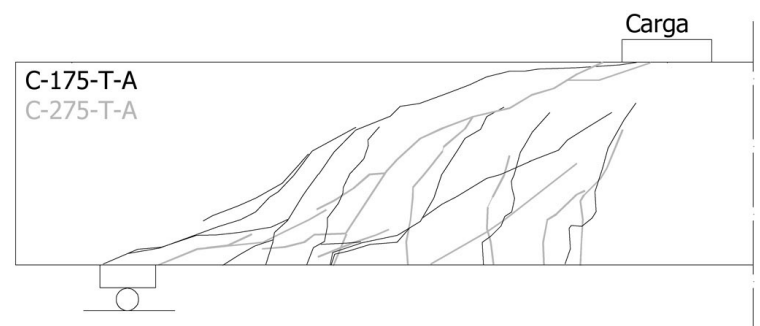

d) cuantía alta armadura longitudinal con estribos

Figura 8. Patrones de fisuración de los ensayos de cortante en rotura. 


\section{PREDICCIONES ANALITICAS SEGÚN DIVERSOS MODELOS}

Tanto para los ensayos de flexión como para los ensayos de cortante, se realizó una predicción analítica de la carga última aplicando la Instrucción EHE-o8 (1) y el Eurocódigo 2 (5), y una predicción numérica del comportamiento de las vigas ensayadas mediante el modelo de análisis no lineal evolutivo CONSHEAR desarrollado por Ferreira (9) (10) a partir del modelo CONS (11), y que considera la deformación por cortante y el estado biaxial de tensiones al evaluar la respuesta estructural del elemento. La formulación del modelo, relativa a la interacción de esfuerzos, se basa en el trabajo previo de los autores recogido en (12). El contenido de finos ha sido modelizado de forma indirecta mediante los parámetros de caracterización mecánica del hormigón. Independientemente del tipo de ensayo, las vigas se simularon mediante elementos de o,1 m de longitud, la sección transversal se discretizó con fibras de 0,011 $\mathrm{m}$ de espesor y la armadura longitudinal con filamentos. La aplicación de la carga se realizó de forma incremental hasta rotura, sin tener en cuenta las ramas de descarga y recarga.

A continuación, se presentan algunos de los resultados más relevantes de las predicciones analíticas y numéricas, en relación a los resultados experimentales.

\subsection{Ensayos a flexión}

La Tabla 3 recoge las cargas últimas experimentales, numéricas y analíticas. Se observa como los valores obtenidos con la
Instrucción EHE-o8 (1) o el EC-2 (5) son siempre inferiores a los experimentales. Esta diferencia es del orden del $13 \%$ para vigas con cuantía alta de armadura longitudinal. Para vigas con cuantía media de armadura longitudinal, la diferencia es del $23 \%$ para vigas con contenido de finos convencional y de $17 \%$ para vigas con contenido alto de finos. En los cálculos con la normativa se han considerado las propiedades mecánicas medidas experimentalmente para cada tipo de hormigón.

Los resultados obtenidos numéricamente son también menores a los experimentales para las vigas armadas con cuantía alta de armadura longitudinal, pero las diferencias son menores que con los valores experimentales ( $5 \%$ ). Para las vigas con cuantía media de armadura longitudinal las predicciones numéricas son prácticamente idénticas, con errores del $2 \%$.

En la Figura 9 se muestra la relación carga-desplazamiento en el centro de la luz para los ensayos de flexión. Los resultados numéricos y experimentales son bastante parecidos. En las vigas con cuantía alta de armadura longitudinal, el instante de inicio de plastificación de la armadura resulta difícil de establecer experimentalmente. En cualquier caso, el agotamiento según el modelo numérico se produce para niveles de carga similares pero bajo desplazamientos mucho mayores en el centro de vano.

La Figura 10 compara los resultados relativos a la deformación en la fibra de la sección centro luz de hormigón situada a la altura de la armadura pasiva traccionada. Se observa una buena correlación de resultados, si bien los valores numéricos de la carga de plastificación son inferiores a los experimentales.

Tabla 3. Cargas últimas experimentales, numéricas y analíticas.

\begin{tabular}{|c|c|c|c|c|c|c|}
\hline & & Exp & nental & & érico & $\begin{array}{c}\text { EHE-o8 } \\
\text { EC-2 }\end{array}$ \\
\hline & & $\underset{(\mathbf{k N})}{\mathbf{P}_{\mathrm{u}}}$ & $\begin{array}{l}\text { Modo de } \\
\text { rotura }\end{array}$ & $\underset{(\mathbf{k N})}{\mathbf{P}_{u}}$ & $\begin{array}{c}\text { Modo de } \\
\text { rotura }\end{array}$ & $\underset{(\mathbf{k N})}{\mathbf{P}_{u}}$ \\
\hline $\mathrm{F} \mathrm{T} \mathrm{A}$ & 175 & 296 & Flexión & 275,6 & Flo & 256 \\
\hline $1-1-1$ & 275 & 290 & Flexión & 261,6 & КТАIOH & 253 \\
\hline T T $\mathrm{M}$ & 175 & 139 & Flexión & 142,7 & & 106,6 \\
\hline $1-1-171$ & 275 & 137 & Flexión & 138,7 & КТАНОН & 113 \\
\hline
\end{tabular}

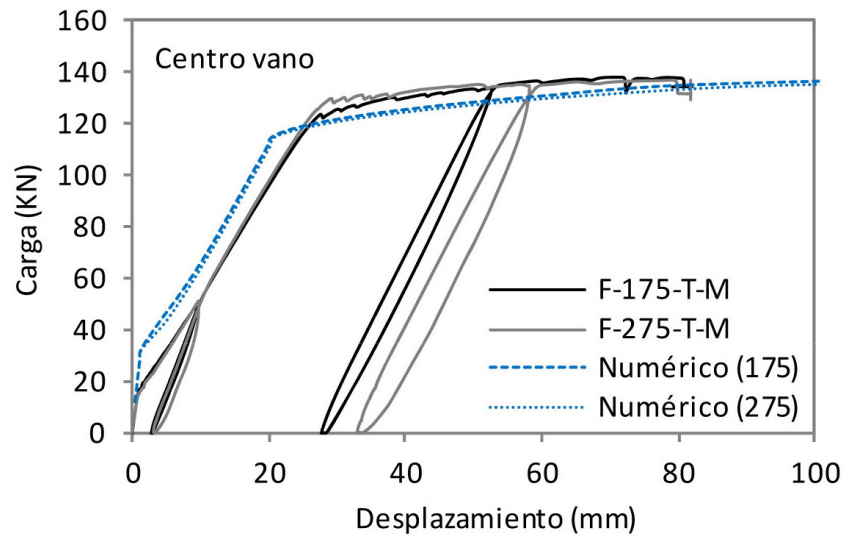

a) cuantía media armadura longitudinal

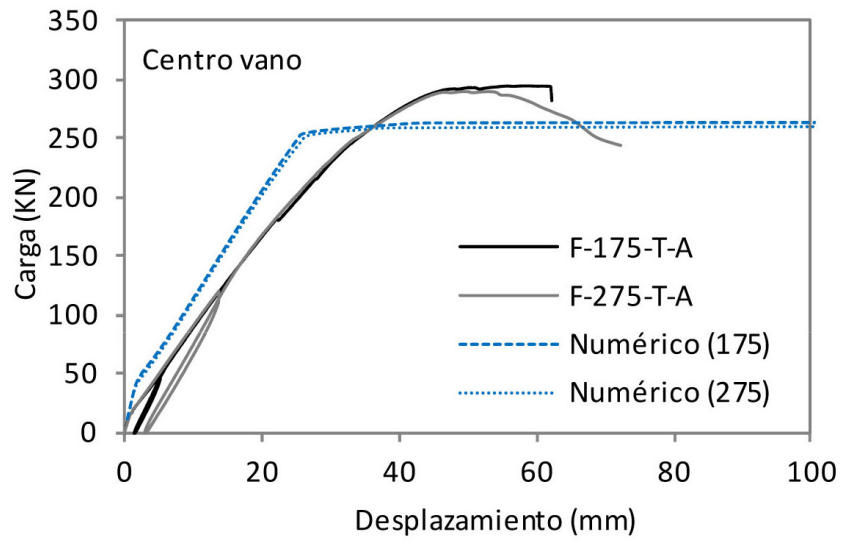

b) cuantía alta armadura longitudinal

Figura 9. Carga-desplazamiento en el centro de la luz, exp vs. num. 


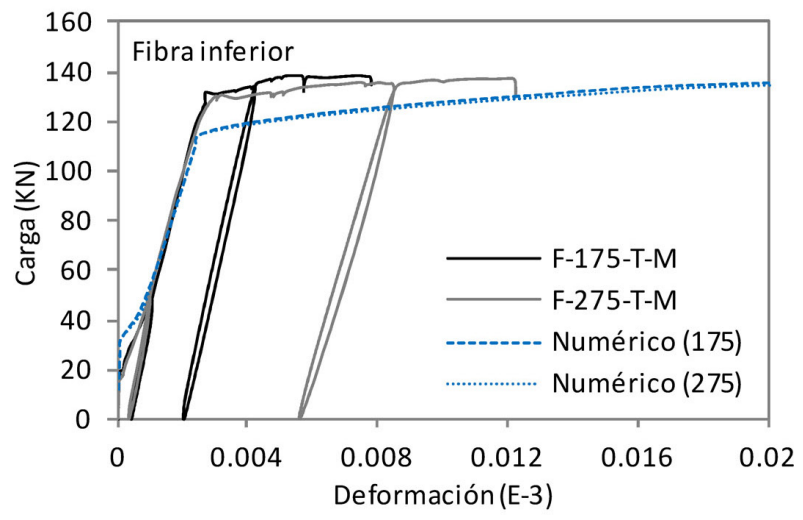

a) cuantía media armadura longitudinal

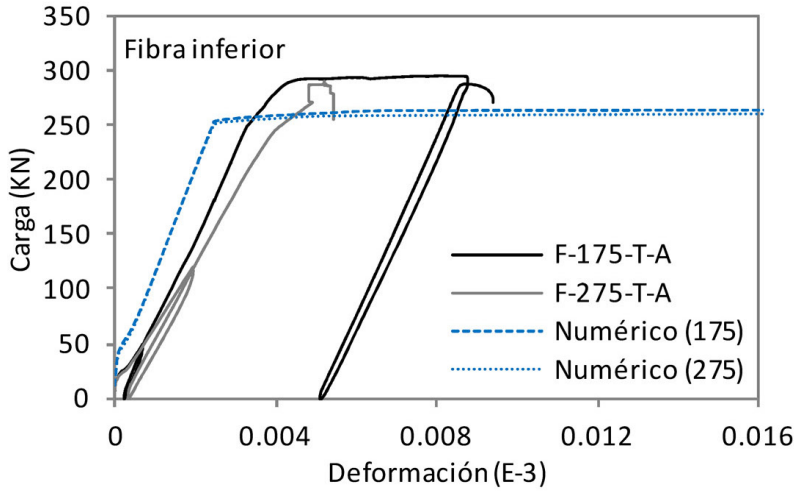

b) cuantía alta armadura longitudinal

Figura 10. Carga-deformación en la fibra a la altura de la armadura longitudinal inferior en la sección centro de la luz, exp. vs num.

\subsection{Ensayos a cortante}

La Tabla 4 recoge las cargas últimas experimentales, numéricas y analíticas obtenidas con la Instrucción EHE-o8 (1) y el EC-2 (5), que coinciden para piezas sin armadura de cortante. Las predicciones analíticas que muestra la Tabla 4 se han obtenido suponiendo un ángulo de inclinación de las bielas igual al obtenido experimentalmente medido a través de los transductores de desplazamiento. Estos resultados son más ajustados que los obtenidos utilizando la formulación general para el cálculo de la inclinación de las bielas, e incluso más exactos que considerando el ángulo obtenido en el instante de fisuración a la altura de la directriz de la pieza de $45^{\circ}$.

La relación experimental y numérica carga-desplazamiento bajo carga se presenta en la Figura 11 para las vigas ensayadas a cortante. En cualquier caso, independientemente de la cuantía de armadura longitudinal y transversal, la respuesta numérica es más rígida que la experimental.

Se observa una discrepancia de rigidez inicial tanto en el modelo flexión como de cortante, que se puede atribuir a incertidumbres en los desplazamientos de los apoyos y la existencia de fisuración previa. El modelo numérico presenta un ajuste aceptable con los resultados experimentales.

La Figura 12 muestra la deformación media de la sección a la altura de la armadura longitudinal inferior y a la altura de la armadura de compresión en una sección situada a 0,6 m del apoyo, donde se ha situado la roseta. Los valores numéricos de deformación máxima son inferiores a los experimentales, en especial para los casos de piezas sin armadura de cortante. Se observa como el modelo es más rígido que el ensayo experimental, especialmente en el caso de la armadura longitudinal inferior. En la zona comprimida, se consigue un mejor ajuste del modelo numérico a las deformaciones experimentales.

La Figura 13 recoge la evolución de la distorsión con la carga aplicada en una sección a o,6 m del apoyo, observándose que las predicciones numéricas se ajustan bastante a los resultados experimentales obtenidos a partir de la roseta. La formación de la fisura inclinada de cortante se corresponde con un incremento brusco de distorsión. Las vigas con armadura transversal desarrollan mayores distorsiones y las vigas sin armadura transversal alcanzan la rotura justo después de la formación de la fisura de cortante, por ello los valores de distorsión son mucho menores. Para las vigas sin armadura transversal con cuantía alta de armadura longitudinal el comportamiento es prácticamente idéntico independientemente del contenido de finos. Para las vigas con armadura transversal, no se verifican diferencias significativas entre los dos contenidos de finos. Destaca nuevamente la dificultad inherente a este tipo de medición local, que es muy vulnerable a la localización de la fisuración. Asimismo, la diferencia en inclinación y posición de las fisuras influye considerablemente en esta medición.

Tabla 4. Cargas últimas experimentales, numéricas y analíticas según la Instrucción EHE-o8 y el EC-2.

\begin{tabular}{|c|c|c|c|c|c|c|c|}
\hline \multirow{2}{*}{\multicolumn{2}{|c|}{$\begin{array}{l}\text { Ensayos } \\
\text { a cortante }\end{array}$}} & \multicolumn{2}{|c|}{ Experimental } & \multicolumn{2}{|c|}{ Numérico } & \multirow{2}{*}{$\frac{\text { EHE-o8 }}{\underset{(\mathbf{k N})}{\mathbf{P}_{\mathrm{u}}}}$} & \multirow{2}{*}{$\frac{\text { EC-2 }}{\mathbf{P}_{\mathbf{u}}}$} \\
\hline & & $\underset{(\mathbf{k N})}{\mathbf{P}_{\mathbf{u}}}$ & $\begin{array}{l}\text { Modo de } \\
\text { rotura }\end{array}$ & $\underset{(\mathbf{k N})}{\mathbf{P}_{\mathrm{u}}}$ & $\begin{array}{c}\text { Modo de } \\
\text { rotura }\end{array}$ & & \\
\hline \multirow{2}{*}{ C-S-M } & 175 & 145,4 & \multirow{2}{*}{ Cortante } & 138,1 & \multirow{2}{*}{ Cortante } & \multicolumn{2}{|c|}{147,5} \\
\hline & 275 & 141,0 & & 132,1 & & \multicolumn{2}{|c|}{139,4} \\
\hline \multirow{2}{*}{ C-S-A } & 175 & 181,1 & \multirow{2}{*}{ Cortante } & 165,7 & \multirow{2}{*}{ Cortante } & \multicolumn{2}{|c|}{174,9} \\
\hline & 275 & 158,4 & & 157,5 & & \multicolumn{2}{|c|}{165,4} \\
\hline \multirow{2}{*}{ C-T-M } & 175 & 220,1 & \multirow{2}{*}{ Cortante } & 224,3 & \multirow{2}{*}{ Cortante } & 214,6 & 91,7 \\
\hline & 275 & 225,0 & & 216,0 & & 213,1 & 96,9 \\
\hline \multirow{2}{*}{ C-T-A } & 175 & 367,3 & \multirow{2}{*}{ Cortante } & 323,0 & \multirow{2}{*}{ Cortante } & 315,9 & 170,1 \\
\hline & 275 & 284,0 & & 252,0 & & 265,9 & 128,1 \\
\hline
\end{tabular}




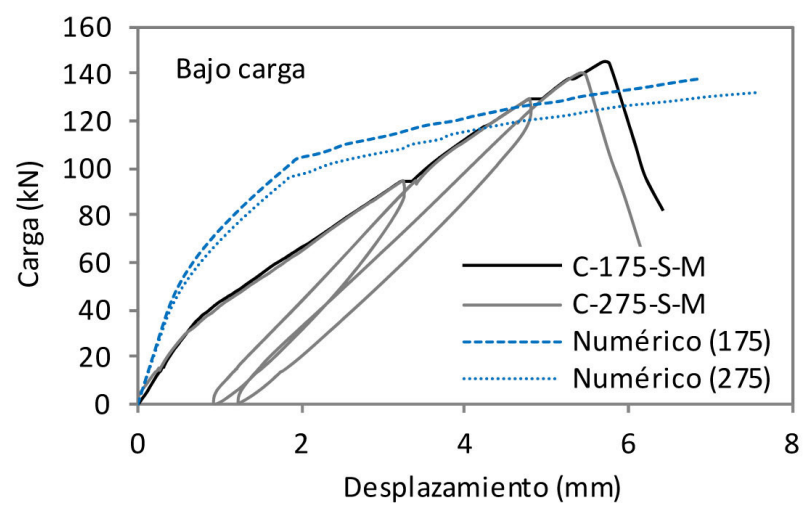

a) cuantía media armadura longitudinal sin estribos

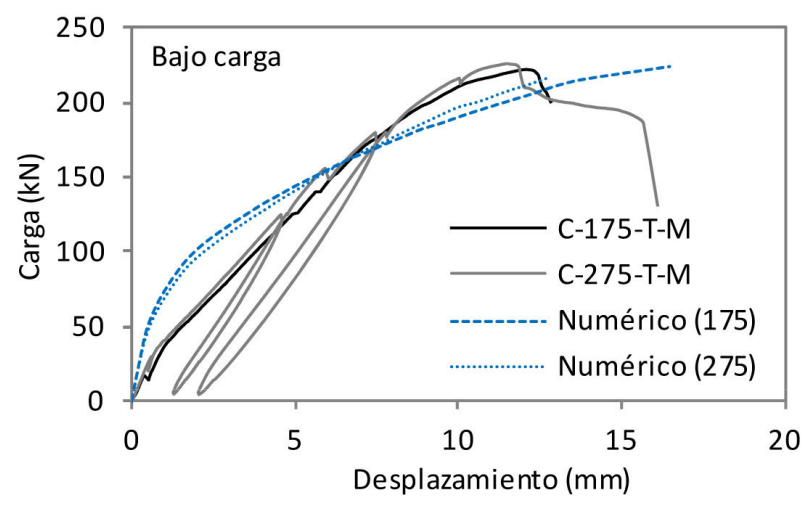

c) cuantía media armadura longitudinal con estribos

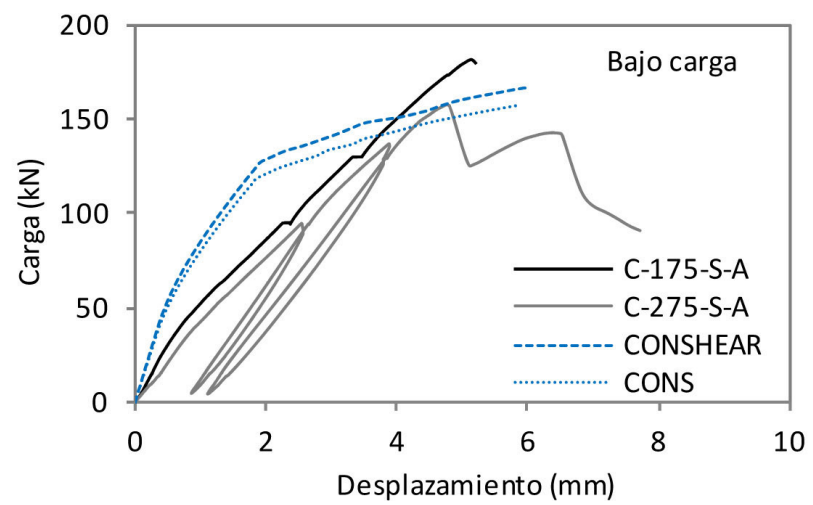

b) cuantía alta armadura longitudinal sin estribos

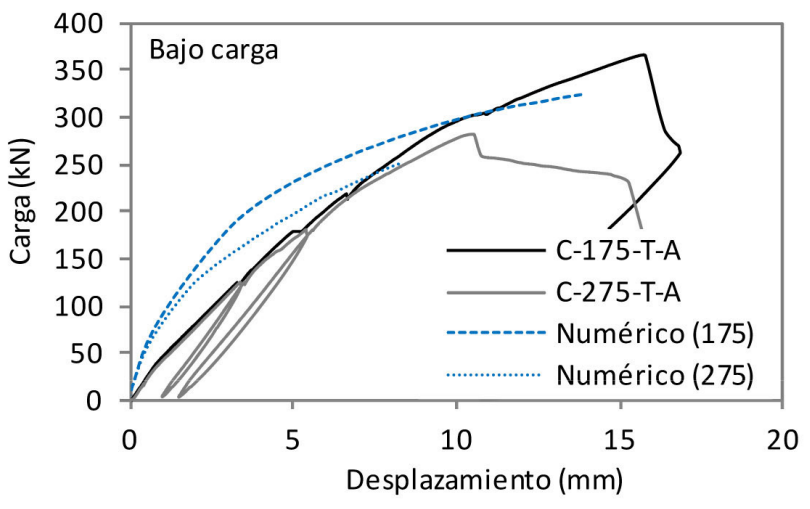

d) cuantía alta armadura longitudinal con estribos

Figura 11. Carga-desplazamiento bajo carga para los ensayos de cortante, experimental vs. numérico.

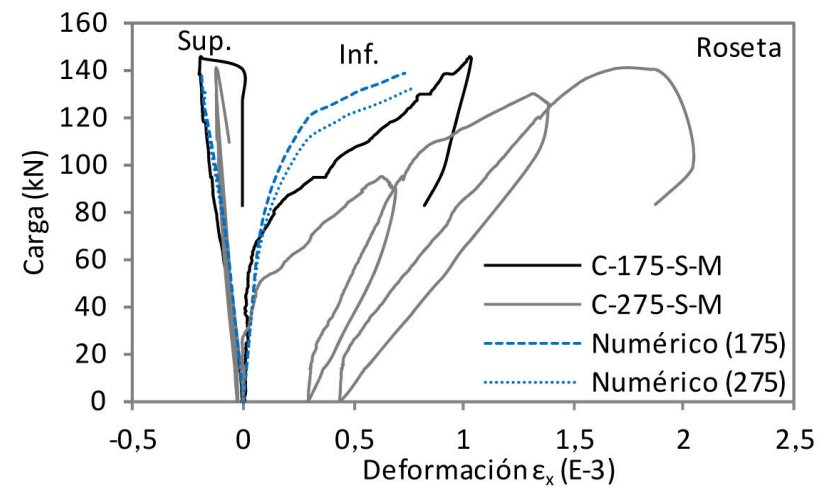

a) cuantía media armadura longitudinal sin estribos

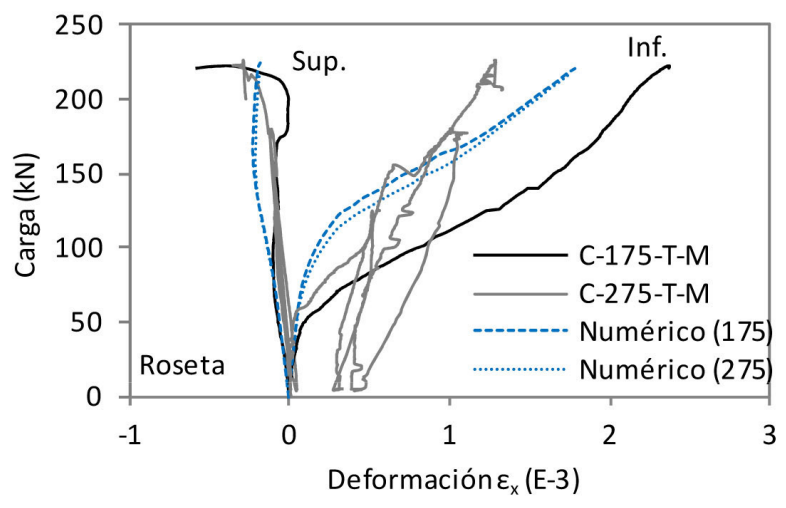

c) cuantía media armadura longitudinal con estribos

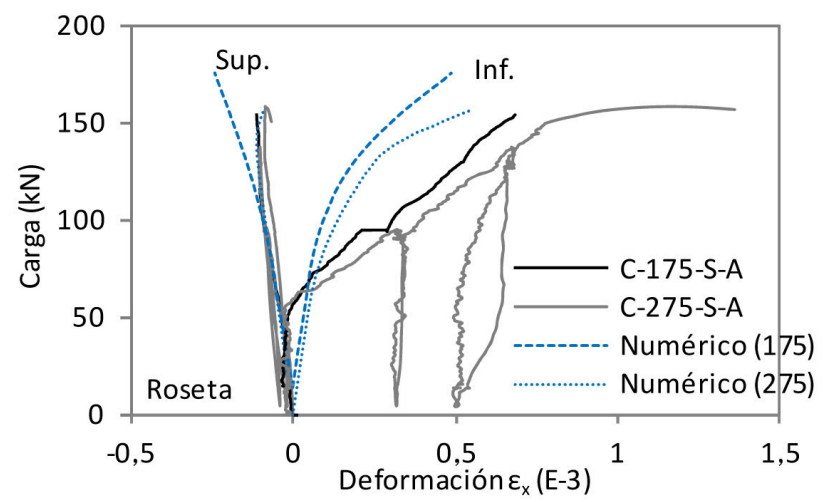

b) cuantía alta armadura longitudinal sin estribos

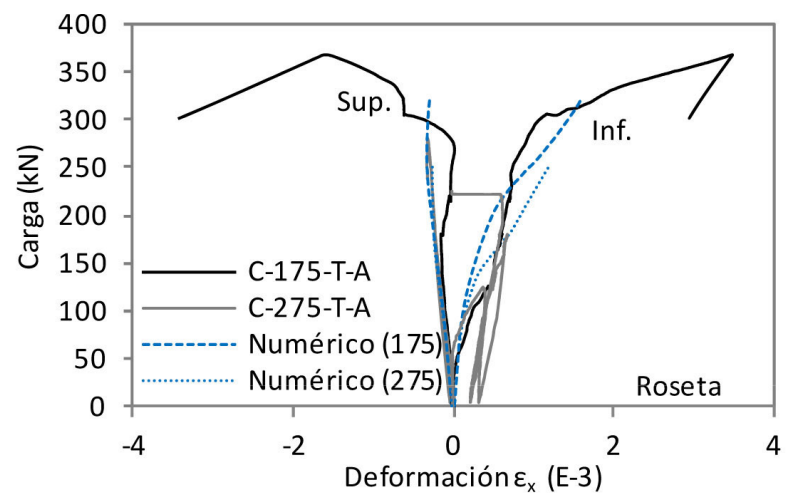

d) cuantía alta armadura longitudinal con estribos

Figura 12. Carga-deformación longitudinal (roseta) los ensayos de cortante, experimental vs. numérico. 


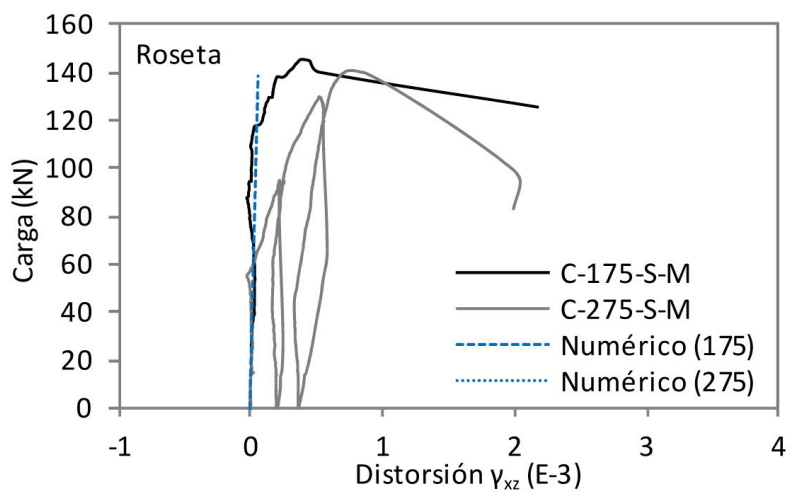

a) cuantía media armadura longitudinal sin estribos

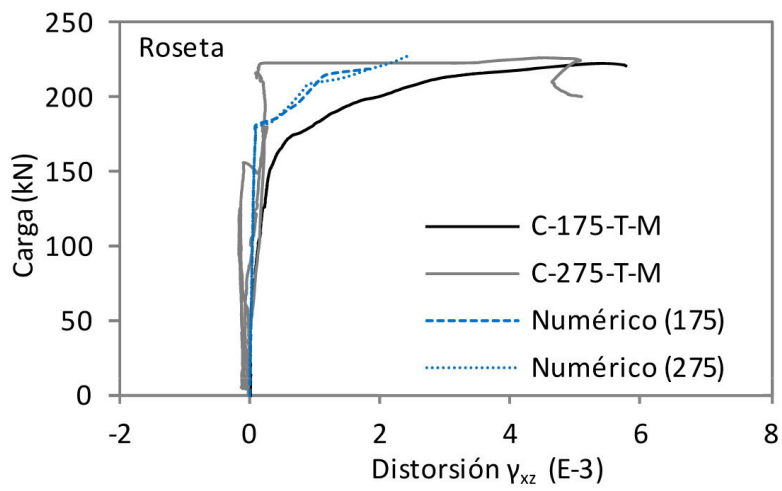

c) cuantía media armadura longitudinal con estribos

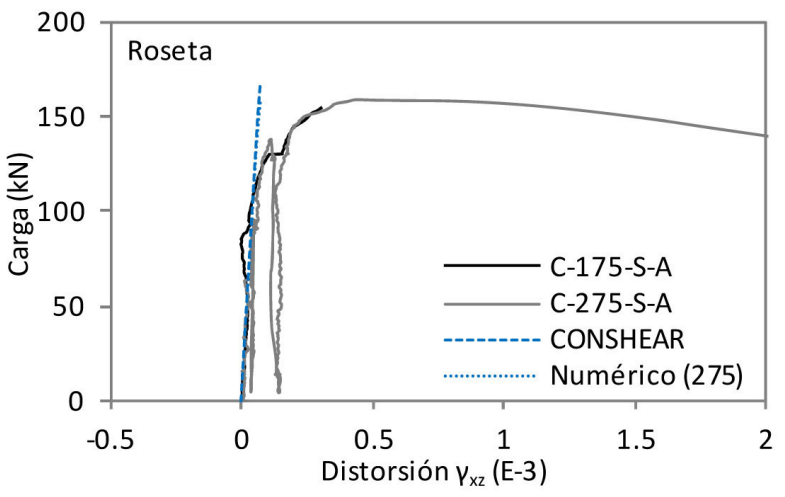

b) cuantía alta armadura longitudinal sin estribos

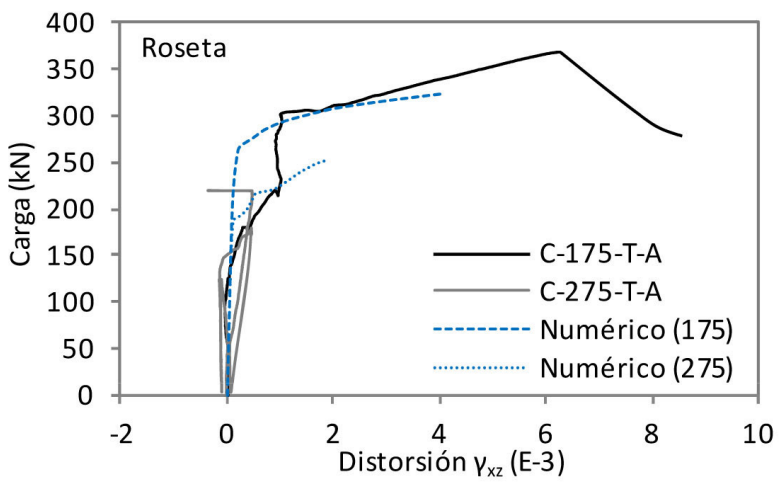

d) cuantía alta armadura longitudinal con estribos

Figura 13. Carga-distorsión (roseta) los ensayos de cortante, experimental vs. numérico.

\section{CONCLUSIONES}

En este trabajo se ha llevado a cabo una campaña experimental sobre vigas de hormigón armado ensayadas a flexión y a cortante, con el objetivo de estudiar la influencia del contenido en finos sobre la respuesta estructural de las mismas. Se han ensayado 8 vigas, agrupadas en 2 series con contenidos de finos de $175 \mathrm{~kg} / \mathrm{m}^{3}$ y $275 \mathrm{~kg} / \mathrm{m}^{3}$. De cada serie, dos vigas se han ensayado a flexión y dos a cortante con cuantías media y alta de armadura longitudinal, con y sin armadura transversal.

Se han realizado predicciones analíticas de la carga última y se ha modelizado numéricamente el comportamiento de las vigas con un modelo de análisis no lineal.

De los resultados obtenidos y de su comparación con las predicciones teóricas cabe extraer las siguientes conclusiones:

Los valores de la resistencia a compresión, resistencia a tracción y módulo de elasticidad del hormigón con alto contenido en finos fueron ligeramente menores que los correspondientes para el hormigón convencional. La reducción máxima de la resistencia a compresión fue del 15,6 \% a los 90 días en la probeta curada en condiciones normalizadas. En cuanto a la resistencia a tracción, la reducción a los 28 días fue del 12,o \%. El módulo de elasticidad sufrió una reducción máxima del 5,5\% en las probetas ensayadas a los 28 días.

El contenido de finos, en el rango estudiado, apenas afecta a la respuesta a flexión. La reducción máxima de carga última es del $2 \%$ en la viga con alta cuantía de armadura longitudinal. Este resultado es coherente con que la resistencia a compresión del hormigón apenas influye en la resistencia a flexión siempre que la rotura sea dúctil. En todos los casos, la carga última experimental es mayor que la carga última teórica y las predicciones numéricas son similares (errores $2-5 \%)$.

La deformabilidad instantánea de las vigas con alto contenido de finos es prácticamente igual a la de las vigas con hormigón convencional. Ello es así a pesar de que las vigas con mayor contenido de finos tienen menor resistencia a tracción y módulo de elasticidad del hormigón.

La fisuración en ambos casos es muy parecida, sin cambios aparentes en el mecanismo resistente, si bien en el hormigón con alto contenido de finos se observaron menor separación de fisuras y menor abertura que en el hormigón convencional, atribuibles a la menor resistencia a tracción.

Las respuestas a cortante se ve más afectada por el aumento de finos que la respuesta a flexión. En general, en los ensayos de cortante, las vigas con $275 \mathrm{~kg} / \mathrm{m}^{3}$ han mostrado una resistencia a cortante menor que las correspondientes vigas con hormigón convencional, en especial para las vigas con cuantía alta de armadura longitudinal y sin armadura de cortante.

La pérdida de capacidad resistente a cortante es tanto mayor a mayor cuantía de armadura transversal $(-22,7 \%$ en la viga C-275-T-A) y de armadura longitudinal $(-12,5 \%$ en la viga 
C-275-S-A). Para cuantías medias apenas se produce reducción de capacidad a cortante (-3,0 \% en la viga C-275-S-M) o incluso aumenta ligeramente $(+2,2 \%$ en la viga $\mathrm{C}-275-\mathrm{T}-\mathrm{M})$. Este hecho se considera debido a la reducción de propiedades mecánicas del hormigón que afecta al cortante que es capaz de resistir el hormigón por fricción a lo largo de la fisura.

Se observa que la carga última experimental que agota las vigas por cortante es superior a la teórica obtenida mediante la Instrucción EHE-o8, en todos los casos, excepto en las vigas sin armadura transversal C-175-S-M y C275-S-A, para las cuales la carga última prácticamente idéntica a la teórica. Las predicciones del EC-2 (5) para piezas con armadura de cortante difieren en mayor medida al no considerar la contribución del hormigón a la resistencia a cortante.

La modelización numérica presenta buenos resultados relativos al comportamiento de carga-desplazamiento, deformación en la armadura longitudinal, distorsiones. Además, el modelo ha mostrado su capacidad para reproducir las dife- rencias de comportamiento entre las vigas fabricadas con uno u otro tipo de hormigón.

Puede extraerse, como conclusión global, que aumentar el contenido de finos de $175 \mathrm{~kg} / \mathrm{m}^{3}$ a $275 \mathrm{~kg} / \mathrm{m}^{3}$ apenas afecta a la resistencia a flexión, deformabilidad o ancho de fisuras y reduce moderadamente la resistencia a cortante, tanto en vigas con armadura transversal como sin ella.

\section{AGRADECIMIENTOS}

Los autores agradecen al Instituto Español del Cemento y sus aplicaciones, la financiación de la campaña experimental desarrollada. Este trabajo se ha desarrollado en el marco del proyecto BIA2012-36848 financiado por el Ministerio de Economía y Competitividad, y cofinanciado con Fondos FEDER. Se agradece a la Fundación Portuguesa para la Ciencia y Tecnología (FCT) la beca de doctorado SFRH/BD/43232/2008, así como a la Generalitat de Catalunya, la beca post-doctoral (ref. 2013 PDJ 0o022) de la primera autora.

\section{REFERENCIAS}

(1) Comisión Permanente del Hormigón, Ministerio de Fomento. (2008). EHE-o8 - Instrucción del hormigón estructural. Madrid: Ministerio de Fomento.

(2) Saéz, C., Aguado, A. (2013). Caracterización mecánica de hormigones con alto contenido de finos. Cemento Hormigón, (955): 50-58.

(3) Katz, A., Baum H. (2006). Effect of high levels of fines content on concrete properties. ACI Materials Journal, 103(6): 474-482, doi: http://dx.doi.org/10.14359/18226.

(4) Vilanova, A., Fernández, I., Agranati, G. (2010). Evaluación de los modelos de predicción del ACI-o8, Eurocódigo 2 y EHE-08, para estimar las propiedades mecánicas del hormigón autocompactante. Informes de la Construcción, 62(520): 43-55, doi: http://dx.doi.org/10.3989/ic.09.069.

(5) CEN. (2002). Eurocode 2: Design of Concrete Structures: Part 1: General Rules and Rules for Buildings. European Committee for Standardization.

(6) ACI Committee 318. (2008). Building Code Requirements for Structural Concrete (ACI 318-o8) and Commentary. Farmington Hills, MI, USA: American Concrete Institute.

(7) Choulli, Y., Marí, A., Cladera, A. (2008). Shear behavior of full-scale prestressed I-beams made with self-compacting concrete. Materials and Structures, 41(1): 131-141, doi: http://dx.doi.org/10.1617/s11527-007-9225-1.

(8) Schiessl, A., Zilch K. (2001). The effects of the modified composition of SCC on shear bond behaviour. In 2nd International Symposium on Self Compacting Concrete. Tokyo.

(9) Ferreira, D., Bairán, J. M. Marí, A. (2013). Numerical simulation of shear-strengthened RC beams. Engineering Structures, 46: 359-374, doi: http://dx.doi.org/10.1016/j.engstruct.2012.06.050.

(10) Ferreira, D., Bairán, J., Marí, A., Faria, R. (2014). Nonlinear analysis of RC beams using a hybrid shear-flexural fibre beam model. Engineering Computations, 31(7): 1444-1483, doi: http://dx.doi.org/10.1108/EC-04-2013-0114.

(11) Marí, A. (2000). Numerical simulation of the segmental construction of three dimensional concrete frames. Engineering Structures, 22(6): 585-596, doi: http://dx.doi.org/10.1016/So141-0296(99)oooo9-7.

(12) Bairán, J. M., Marí, A. (2007). Shear-bending-torsion interaction in structural concrete members: A nonolinear coupled sectional approach. Archives of Computational Methods in Engineering, 14(3): 249-278, doi: http://dx.doi. org/10.1007/s11831-007-9007-5. 